\title{
Coupled Registration-Segmentation: Application to Femur Analysis with Intra-subject Multiple Levels of Detail MRI Data
}

\author{
Jérôme Schmid ${ }^{\star}$, Jinman Kim, and Nadia Magnenat-Thalmann \\ MIRALab, University of Geneva, Switzerland \\ \{schmid,jinman.kim, thalmann\}@miralab.ch
}

\begin{abstract}
The acquisition of intra-subject data from multiple images is routinely performed to provide complementary information where a single image is not sufficient. However, these images are not always coregistered since they are acquired with different scanners, affected by subject's movements during scans, and consist of different image attributes, e.g. image resolution, field of view (FOV) and intensity distributions. In this study, we propose a coupled registration-segmentation framework that simultaneously registers and segments intra-subject images with different image attributes. The proposed coupled framework is demonstrated with the processing of multiple level of detail (LOD) MRI acquisitions of the hip joint structures, which yield efficient and automated approaches to analyze soft tissues (from high-resolution MRI) in conjunction with the entire hip joint structures (from low resolution MRI).
\end{abstract}

Keywords: Segmentation; Registration; Deformable models; Hip joint; Level of detail.

\section{Introduction}

The acquisition of intra-subject data from multiple images is routinely performed to provide complementary information where a single image is not sufficient. As an example, the acquisition of MRI images of the hip joint at multiple levels of detail (LOD) is often used to identify bone changes and soft tissue abnormalities, which might explain the development of arthritis. In this protocol, exemplified in Fig. 2, a low resolution MRI that covers a large field of view (FOV) is acquired to image the whole hip joint structures (i.e. entire femur bone), whereas a high resolution MRI with limited FOV and centered at the hip joint is acquired to reveal the fine details of the soft tissues. Similarly, the use of multiple images of joints acquired in different postures was demonstrated to be an effective approach for biomechanical motion analysis, which required these images to be aligned (e.g., [1]). From these examples, there is a crucial need for image registration where multiple acquisitions are not always co-registered. In addition, the segmentation

\footnotetext{
^ Corresponding author.
} 
of the structures of interest (SOIs), shared among the acquisitions, is commonly required for quantitative correspondence.

The ability for concurrent segmentation and registration has been demonstrated to be a powerful method in recent years. A seminal paper by Yezzi et al. 2] presented a variational framework for simultaneous segmentation and registration using deformable models, and gave birth to a variety of methods 3 4 4 5 6 6 7 with extensions such as the support for more than 2 images or nonrigid transforms. In other studies, voxel-based approaches, formulated as a maximum a posteriori (MAP) estimation problem were proposed [8]. Atlas-based methods [5] were also presented, but were limited to data with atlas availability. Further, atlas-based methods generally involved non-rigid transforms, and were thus not appropriate to e.g. a rigid structure imaged in different positions.

In this paper, we propose a coupled registration-segmentation framework based on deformable models for intra-subject images acquired with different protocols. The framework is able to handle intra-subject images characterized by varying FOV in which SOIs can be partially visible and thus have a limited correspondence which seriously complicates their direct registration. Further, our framework is atlas-free, does not limit the number of images or SOIs, and does not restrict the nature or evolution strategies of the deformable models. Its effectiveness is demonstrated in the registration-segmentation of femurs in intrasubject MRIs acquired at multiple LODs (hereon referred to as "multiple LOD MRIs"). Our coupled framework will simultaneously segment and register the multiple LOD MRIs, and thus enable an efficient and automated approach to the analysis of (pathological) soft tissues from images of the joint (high-resolution) in conjunction with the entire hip structure (low-resolution).

\section{Coupled Registration-Segmentation}

\subsection{Framework Overview}

In this work we propose to segment and register the same SOIs in $N$ images $I^{1}, \ldots, I^{N}\left(I^{i}: \Omega^{i} \rightarrow \Re, \Omega^{i} \in \Re^{D}(D=2,3)\right)$. For the sake of brevity, we only present our methodology in case of a single SOI, while the proposed approach can be easily extended to multiple SOIs. We assume that the segmentation is achieved by using $N$ deformable models (DM) represented by shapes $S^{1}, \ldots, S^{N}$, composed of a same number $n$ of points $x_{j}^{i}: S^{i}=\left\{x_{1}^{i}, \ldots, x_{n}^{i}\right\}$. No restrictions are made on the nature of the DM (e.g., curves [4], simplex meshes [10]), except that their shapes must share the same number of points and be in point correspondence. Similarly, DMs can adopt any type of evolution strategies (e.g., Gradient flow [2], Newtonian law equations [11]) as long as they can be expressed as an update operator $\Psi\left(S^{i}\right)$, which returns new state of the shape at each evolution step.

The registration consists in computing the mappings $g^{i \leftarrow j}$ to transform $S^{j}$ to $S^{i}$. Similarly to [7, instead of computing the $N^{2}$ possible mappings $g^{i \leftarrow j}$, we estimate the mappings $g^{i}$ that transform a common shape $\bar{S}$ to each shape $S^{i}$. In 
case of linear transforms and shapes in point correspondence, we propose to express these mappings $g^{i}$ by using a generalized Procrustes alignment (GPA) [12]. During this procedure, mean shape $\bar{S}=\left\{\overline{x_{1}}, \ldots, \overline{x_{n}}\right\}$ and mapping $g^{i}$ estimates are iteratively updated. The mappings are estimated in a least square sense:

$$
g^{i}=\underset{g}{\operatorname{argmin}} \sum_{j}\left\|x_{j}^{i}-g\left(\bar{x}_{j}\right)\right\|^{2}=\underset{g}{\operatorname{argmin}} \sum_{j} d_{g}\left(x_{j}^{i}\right)^{2} .
$$

An overview of the steps involved in our coupled registration-segmentation framework is depicted in Fig. 1] in which segmentation and registration are used in an interleaved manner. The steps are repeated until segmentations convergence. First shapes $S^{i}$ are updated given the segmentation update operator $\Psi$. Then the resulting shapes $\Psi\left(S^{i}\right)$ are aligned with the GPA. The final stage performs a linear "blending" at the point level between shapes $\Psi\left(S^{i}\right)$ and the back-transformed mean shapes $g^{i}(\bar{S})$ to get new shapes $S^{\prime i}$ :

$$
S^{i}=\lambda g^{i}(\bar{S})+(1-\lambda) \Psi\left(S^{i}\right) .
$$

Parameter $\lambda \in[0,1]$ is used as a stiffness coefficient, which expresses the degree of constraint applied by the registration to the segmentation. Theoretically, if shapes only differ by a linear transform $\lambda$ should be set to 1 . However, in practice it is better to slightly relax $\lambda$ at the beginning of the segmentation. This provides more freedom to the segmentation, especially when the shapes are not initialized closely enough and they need to be significantly deformed. In this paper, we linearly increases $\lambda$ from 0.7 to 1 .

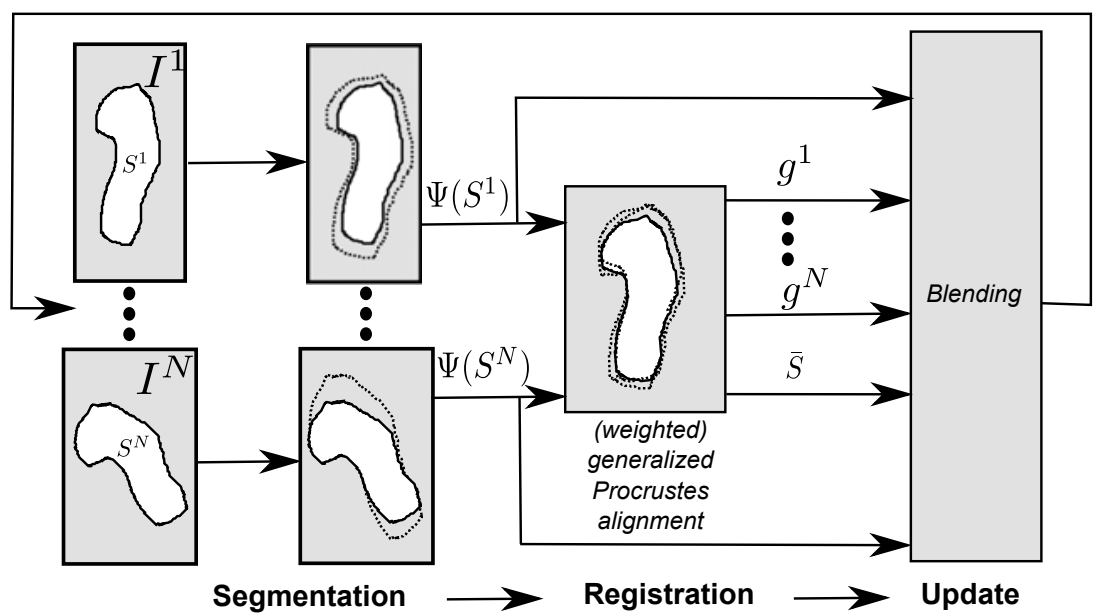

Fig. 1. Coupled registration-segmentation step: initially, a segmentation step is executed, evolving $N$ shapes $S^{i} \rightarrow \Psi\left(S^{i}\right)$; resulting shapes $\Psi\left(S^{i}\right)$ are then aligned with a (weighted) GPA to yield alignment mappings $g^{i}$ and mean shape $\bar{S}$ ); finally, shapes $\Psi\left(S^{i}\right)$ and back-transformed mean shapes $g^{i}(\bar{S})$ are blended to produce new shapes 


\section{$2.2 \quad$ Weighting Procedure}

A major issue with the aforementioned step is that all shape points are equally treated in the registration. If these points are invalid from a segmentation viewpoint (e.g. point not lying on organ boundary or out of the image FOV), they will corrupt the registration. As a result, bad estimates of the mean $\bar{S}$ and mappings $g^{i}$ will be computed. Hence we apply a simple yet efficient solution that uses a weighted GPA. Given weights $w_{j}^{i}$ associated with point $x_{j}^{i}$ of shape $S^{i}$, the weighted alignment is obtained by weighting (i) the mean (i.e. $\left.\bar{x}_{j}=\sum_{i} w_{j}^{i} x_{j}^{i} / \sum_{i} w_{j}^{i}\right)$ and (ii) the least square minimization in Eq. (1), by replacing the distance terms $d_{g}\left(x_{j}^{i}\right)$ with $w_{j}^{i} d_{g}\left(x_{j}^{i}\right)$.

In this paper, we express the weight $w_{j}^{i}$ as the sum of "reliability" terms $F^{k}\left(\theta\left(x_{j}^{i}\right)\right): w_{j}^{i}=\sum_{1 \leq k \leq N} F^{k}\left(\theta\left(x_{j}^{i}\right)\right)$. The quantity $\theta\left(x_{j}^{i}\right)=\theta_{j}^{i}$ is a features vector computed from the image $I^{i}$, the shape $S^{i}$ and the point $x_{j}^{i}$. The intrareliability term $F^{i}\left(\theta_{j}^{i}\right)$ accounts for the segmentation quality at $x_{j}^{i}$ in image $I^{i}$. Similarly, inter-reliability terms $F^{k}\left(\theta_{j}^{i}\right), k \neq i$ additionally consider the coupling with other images. These terms depend on the segmentation strategy and available prior knowledge (see next Section for examples). If we consider (i) the features vector $\theta_{j}^{i}$ as the observation of a random variable $\Theta$, (ii) the event $w$ " $\theta_{j}^{i}$ is the features vector obtained in case of optimal segmentation at $x_{j}^{i}$ ", and (iii) $F^{k}\left(\theta_{j}^{i}\right)=p^{k}\left(w \mid \theta_{j}^{i}\right)$ as a posteriori probability function, $w_{j}^{i}$ computation is analog to a "sum rule" commonly used in multi-classifiers approaches.

\section{Coupled MRI Bone Registration-Segmentation}

Our experiments are related to the hip joint image analysis to detect abnormal bone shape (changes) that may yield the formation of arthritis [13. We further suggest that the automated alignment of multiple LOD MRIs, together with its segmentation will better support clinical diagnosis and biomechanical studies. We adopted our previous MRI bone segmentation approach 11], which uses DMs that are semi-automatically initialized and driven by dynamic law equations. The evolution is coupled with shape priors expressed as multi-resolution statistical shape models (SSM). Convergence is obtained when shapes variations between two evolution steps are small with respect to a chosen threshold. Image forces used in the DM evolution are based on gradient directions and on the normalized cross correlation ratio $N C C\left(q_{j}^{i}, r q_{j}^{i}\right)$ between intensity profiles [10] $q_{j}^{i}$ extracted at each point $x_{j}^{i}$ and reference profiles $r q_{j}^{i}$. When the segmentation is near completion, the effects of shape priors are turned off enabling the segmentation of fine details not captured by the SSM.

Two acquisition protocols VIBE (VB) and TrueFISP (TF) were used to get the low $\left(1.3 \times 1.3 \times 5 \mathrm{~mm}^{3}\right.$, Fig. $\left.2 \mathrm{a}\right)$ and high $\left(0.6 \times 0.52 \times 0.52 \mathrm{~mm}^{3}\right.$, Fig. $\left.2 \mathrm{~b}\right)$ resolution MRIs, respectively. As depicted in Fig. 2, their FOV differ significantly where the TF focuses on the joint while the VB covers the full femur bone. The computation of weights of Sec. 2.2 is critical. Weights are constantly updated 
during the segmentation as follows: shape points out of their corresponding image FOV have null weights (lowest weight), otherwise the NCC ratio is used to define the intra-reliability $F^{i}\left(\theta_{j}^{i}\right)$ and inter-reliability $F^{k}\left(\theta_{j}^{i}\right)$ as $N C C\left(q_{j}^{i}, r q_{j}^{i}\right)$ and $N C C\left(q_{j}^{i}, q_{j}^{k}\right)$, respectively. By giving higher weights to a shape point whose IP is similar to the other shapes' IPs, robust estimates of the mean shape and alignment transforms are obtained. This provides thus an effective way to express point reliability. The NCC is invariant to linear intensity changes, thus being useful for our MRIs acquired with different protocols.

\section{Results}

Fifteen volunteers were scanned based on Sec. 3 imaging protocols. For each subject, multiple LOD MRIs (TF and VB) were processed with our proposed coupled registration-segmentation framework. For comparative analysis, the VB was also segmented using the same deformable model as in the coupled approach. This is referred to as a single segmentation. The VB image was segmented instead of the $\mathrm{TF}$ in order to have a more reliable and objective estimate since in [11] images with small FOV were not used. For quantitative analysis, the Dice's similarity coefficient (DSC) was used to compare the automated femur segmentation results with that of manual delineations (as the ground truths) defined by experienced researchers under the supervision of a radiologist. A DSC of 1.0 was given to segmentation results having identical overlap to the ground truth. In the first experiment, multiple LODs of $\mathrm{VB}$ and $\mathrm{TF}$ were used, in which the
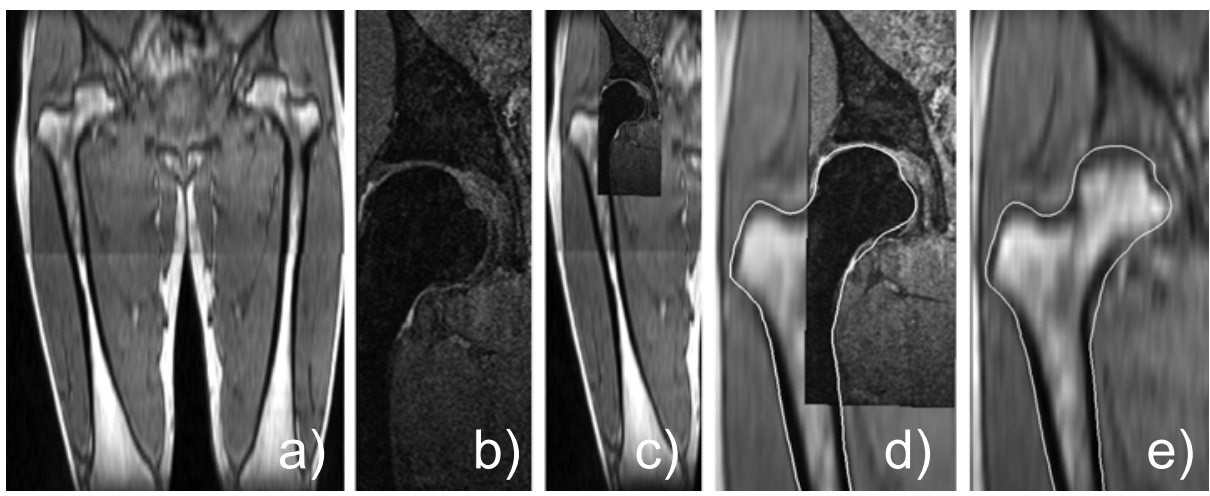

Fig. 2. a) VB and b) TF images of a same subject acquired at different LODs and positions. The higher LOD of the TF is clearly able to image the cartilages and the femoral head in greater details than with the VB. On the other hand, VB provides full imaging of the femur. These multiple LOD images offer a unique possibility to simultaneously analyze in correspondence, the full femur together with the fine detailed soft tissue structures. The alignment resulting from our coupled approach is shown in c) with corresponding white segmentation contours overlaid in d). In comparison, single segmentation results of a) are shown in e). 

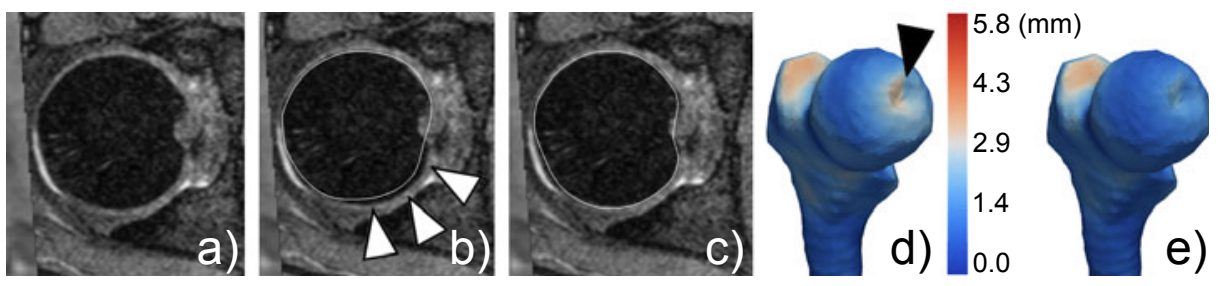

Fig. 3. a) A close-up transversal view of a femoral head visible in the TF. Results from b) single and c) coupled segmentation are overlaid as white contours. The white arrows indicate poorly segmented areas in b) which are markedly corrected in c) using the additional information from the TF MRI. Average distance errors computed from the single d) and coupled e) segmentation results are mapped on an arbitrary femur shape. The black arrow indicates the Fovea Capitis for which larger error differences are observed.

subjects' leg exhibited low flexion/abduction/adduction amplitudes (i.e. close to the "neutral" position) as shown in Fig. 2a) and b).

The result of our coupled approach is shown in Fig. 2r) and d), where both LOD MRIs have been automatically aligned (superimposed together) and segmented, respectively. Here, we can see that the alignment appears to be satisfactory and the segmentation shape correctly contours the bone in both VB and TF MRIs. The surrounding soft tissues, i.e. muscles, were slightly misaligned, although visibly accurate. This was expected since the alignment was based on a rigid mapping of the femur bone and thus did not take into account the soft tissue deformations. In Fig. 22), the segmentation result using only the VB is presented. Visual differences to the coupled approach results are very subtle. The visual findings are validated with the average DSC measures of $0.917 \pm 0.03$ and $0.925 \pm 0.01$ for the single and coupled segmentations, respectively.

Although the improvements with the coupled approach are small for the entire femur segmentation ( $\approx 1 \%$ increase in DSC), the coupled approach is markedly more accurate in the segmentation of the finer details that were only available in the TF (Fig. 3) . A distance error (in mm) was calculated between the ground truth and the segmentation results for both the single (Fig. $3 \mathrm{~d}$ ) and the coupled (Fig. 3.) approaches, averaged among all the subjects, and finally mapped on an arbitrary reference shape. These distance errors better reveal that the finer information from the TF contributed to a better segmentation (e.g., fine details of the fovea capitis). In this first experiment, the position of the leg was neutral for both the VB and the TF MRIs. However, in clinical practice, it often happens that stronger alignment differences between the image acquisitions are observed due to patient movement between the scans. Large leg rotation patterns make the alignment more challenging and locally affect the intensity distribution around the bones. In order to analyze the capability of our coupled framework in the presence of large movement between the scans, we gathered 15 additional datasets of the same subjects performing large leg rotation patterns during the TF acquisition, as shown in Fig. 4. 

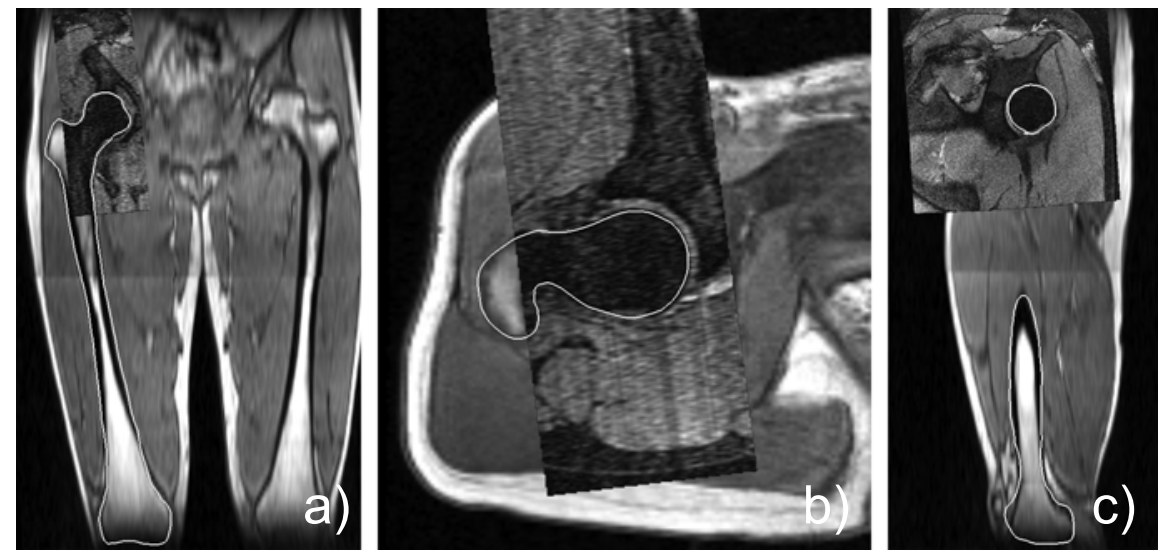

Fig. 4. Segmentation and alignment result of a multiple LOD MRIs of VB and TF shown in a) coronal b) transversal and c) sagittal view slices. In this example, the subject performed a large rotation movement of the leg.

These figures illustrate the strong misalignment that needed to be recovered between the bone extracted in the VB and the TF MRIs. As shown in Fig. 4, the registration of the femur performed well, where we can clearly see the shape outline of the femur between the VB and the TF. Similarly to the previous experiments (Fig. 2), the quantitative measures of the femur segmentation were almost identical (DSC of $0.917 \pm 0.03$ and $0.924 \pm 0.01$ for single and coupled approaches respectively). This suggests that our framework is robust in regards to the mis-alignment orientation of the LOD MRIs as long as these MRIs share a common SOI. We conducted further tests to evaluate our coupled approach in the case where more than two LOD MRIs were available. When our framework was applied to multiple LOD MRIs consisting of a VB and two TF (neutral and large movement), all three images were successfully aligned and the segmentation accuracy was in line with all the other experiments (alone and coupled DSC results of $0.917 \pm 0.03$ and $0.929 \pm 0.01$, respectively).

\section{Discussion and Conclusion}

This study presented a framework for simultaneous registration and segmentation of intra-subject multi-modal images. We exemplified our framework with the application to multiple LOD MRIs. The experimental results demonstrated that our framework was able to robustly align the multiple LOD MRIs that were different in resolution and FOV. At the same time, the combined information in the multiple LOD MRIs was exploited in the segmentation, thus improving the results when compared to the segmentation using a single MRI. We measured our segmentation results to those derived from manual delineation and found the results to be highly accurate. Since the accuracy of the registration is dependent 
on the segmentation in our coupled approach, we suggest that our registration is equally accurate. With our coupled registration-segmentation framework, high and low resolution images of the hip joint structures were simultaneously aligned and segmented, thus offering quantitative correspondence for use in e.g. clinical diagnosis and biomechanical analysis. Current effort is put in automating the initialization of the DMs. Our future work will involve the evaluation of our coupled framework to a wider variety of imaging modalities as well as the extension to non-rigid transforms.

Acknowledgments. This work was supported by the 3D Anatomical Human Project (MRTN-CT-2006-035763) funded by the European Union. We thank our partners, the University Hospital of Geneva and the Great Theater of Geneva.

\section{References}

1. McPherson, A., Kärrholm, J., Pinskerova, V., Sosna, A., Martelli, S.: Imaging knee position using MRI, RSA/CT and 3D digitisation. J. Biomech. 38(2), 263-268 (2005)

2. Yezzi, A., Zollei, L., Kapur, T.: A variational framework for joint segmentation and registration. In: Proc. IEEE MMBIA, pp. 44-51 (2001)

3. Moelich, M., Chan, T.: Joint segmentation and registration using logic models. Technical Report CAM Report 03-06, UCLA (2003)

4. Unal, G., Slabaugh, G.: Coupled PDEs for non-rigid registration and segmentation. In: Proc. CVPR, vol. 1, p. 168. IEEE Computer Society Press, Los Alamitos (2005)

5. Wang, F., Vemuri, B.C.: Simultaneous registration and segmentation of anatomical structures from brain MRI. In: Duncan, J.S., Gerig, G. (eds.) MICCAI 2005. LNCS, vol. 3749, pp. 17-25. Springer, Heidelberg (2005)

6. Droske, M., Rumpf, M.: Multiscale joint segmentation and registration of image morphology. IEEE Transactions on Pattern Analysis and Machine Intelligence 29(12), 2181-2194 (2007)

7. Lord, N., Ho, J., Vemuri, B.: USSR: A unified framework for simultaneous smoothing, segmentation, and registration of multiple images. In: Proc. ICCV, pp. 1-6 (2007)

8. Wyatt, P., Noble, J.: MAP MRF joint segmentation and registration of medical images. Med. Image Anal. 7(4), 539-552 (2003)

9. Pohl, K.M., Fisher, J.W., Levitt, J.J., Shenton, M.E., Kikinis, R., Grimson, W.E.L., Wells, W.M.: A Unifying Approach to Registration, Segmentation, and Intensity Correction. In: Duncan, J.S., Gerig, G. (eds.) MICCAI 2005. LNCS, vol. 3749, pp. 310-318. Springer, Heidelberg (2005)

10. Delingette, H.: General Object Reconstruction Based on Simplex Meshes. Int. J. Comput. Vis. 32(2), 111-146 (1999)

11. Schmid, J., Magnenat-Thalmann, N.: MRI Bone Segmentation using Deformable Models and Shape Priors. In: Metaxas, D., Axel, L., Fichtinger, G., Székely, G. (eds.) MICCAI 2008, Part I. LNCS, vol. 5241, pp. 119-126. Springer, Heidelberg (2008)

12. Gower, J.: Generalized Procrustes analysis. Psychometrika 40, 33-51 (1975)

13. Evangelisto, A., Wakefield, R., Emery, P.: Imaging in early arthritis. Best Pract. Res. Clin. Rheumatol. 18(6), 927-943 (2004) 